\title{
Discrete Type SIR Epidemic Model with Nonlinear Incidence Rate in Presence of Immunity
}

\author{
TAHERA PARVIN \\ Mathematics Discipline \\ Khulna University Khulna-9208, \\ BANGLADESH. \\ PANKAJ KUMAR MONDAL \\ Mathematics Discipline \\ Khulna University Khulna-9208, \\ BANGLADESH.
}

\author{
ARIFUL ISLAM \\ Mathematics Discipline \\ Khulna University Khulna-9208, \\ BANGLADESH. \\ MD. HAIDER ALI BISWAS \\ Mathematics Discipline \\ Khulna University Khulna-9208, \\ BANGLADESH.
}

\begin{abstract}
Mathematical modeling is very important to describe the dynamic behavior of biological and biomedical systems. The SIR model is the most common mathematical model of epidemics. An epidemic occurs if the number of people infected with a disease is increasing in a population. A numerical discretization for an SIR epidemic model is discussed, where the incidence rate is assumed to be Beddington-DeAngelis type. In particular, we reconsider a SIR epidemic model with Non Linear incidence and treatment rate derived by (Dubey et al. 2015) [1]. We applied Euler method to discretize this model. This discretization leads to a numerical scheme which can be considered as a discrete system. Then we analyzed the dynamics of the obtained discrete system. We developed the model with the focus on the concentration of the basic reproduction number and related stability analysis for the disease-free and endemic equilibrium points. Finally, We have performed numerical simulations to illustrate the disease behavior.
\end{abstract}

Keywords - discrete SIR model, Beddington-DeAngelis type nonlinear incidence rate, Euler method, basic reproduction number, stability.

Received: March 7, 2020. Revised: September 30, 2020. Accepted: October 12, 2020. Published: October 24, 2020.

\section{Introduction}

Mathematical modeling is playing an incredible role for providing quantitative insight into multiple fields. It has already contributed to a better understanding of the mechanisms of various diseases. Mathematical modeling has gotten attention because modeling and simulation of any physical phenomena allows us for rapid assessment. So it is mainly used to describe the real phenomena which lead to design better prediction, management and control strategies.Infections and infectious diseases are massive burden on many societies, including the countries. Mathematical models narrating the population dynamics of infectious diseases have been playing an important role in better understanding epidemiological patterns and disease control for a long time. In many cases a simple mathematical model can reveal the nature of the infectious disease transmission, which plays an significant role in the control and prevention of the infectious diseases. Mainly Differential equations and difference equations are two allegory mathematical approaches to modeling epidemic dynamical systems. Fortunately, mathematical models are uniquely positioned to provide a tool amenable for rigorous analysis, hypothesis generation, and connecting results from isolated in vitro experiments with results from in vivo and whole organism studies. In particular, one of the most successful combinations of expertise is that of experimental and biological sciences with the mathematical and computational sciences. The use of mathematical models was proven to be fundamental towards advancing physics in the 20th century, and many are projecting mathematics to play a similar role in advancing biological discovery in the 21 st century. Mathematical and computational models have already begun to play an increasingly large role in the advancement of biological and biochemical research because they make it possible to quantitatively bridge the gap between data 
gathering and mechanism testing by providing a set of analytical and numerical tools.

The birth of mathematical theory of epidemics can be described to the work of Daniel Bernoulli in 1760 [2], who developed a discrete epidemic model to analyze the mortality of smallpox, and more recently to Sir Ronald Ross [3], Anderson Gray McKendrick [4], and the statistician William Ogilvy Kermack. Sir Ronald Ross, an English physician and Nobel Laureate, developed the first mathematical models for the study of the transmission dynamics of malaria [3]. McKendrick and Kermack published a series of papers, introducing a deterministic epidemiological model and their celebrated threshold theorem [4-6]. In the first paper of this series a discrete-time epidemic model is considered, which leads to a continous-time model as the time steps are taken to the limit. After the 2003 SARS outbreaks, and more recently the 2009 H1N1 pandemic and the 2014 Ebola epidemics, efforts to connect models to data have increased greatly. Brauer developed a discrete-time model for SARS [7]. And many other epidemics of particular diseases have also been modeled in a discrete framework. These include measles [8], tuberculosis [9], rodent-hantavirus [10, 11], chytridiomycosis in amphibians [10], plant diseases [12], and diseases involving vectorhost transmission [13] and vertical transmission [14].

The connection between epidemic models in discrete-time and continuous-time settings has been observed in the past. For example, Pellis et al. [15] examined and extended the insights that can be gained from Ludwig's result [16]. Biswas et al., 2020 investigated and analyzed the transmission of most devastating infectious diseases independently in which mathematical modeling was the key tool [17] (see also Biswas et al., 2014 [18]) . At present, there has been an increasing benefit on discrete population dynamical systems because of their rich dynamics behavior and suitability. On the one hand, the discrete models display richer dynamical behavior than the continuous models, which brings more challengeable problems for researches.
In theoretical epidemiology the Euler discretization has been extensively utilized to build the discrete epidemic models [ 19-21]. Chinviriyasit et al. discussed a SEIRS model [34]. Henneman et al. formulated a mathematical modeling of influenza and a secondary bacterial infection [35]. The main idea is discretizing the existing continuous models. The main focus of our study is to discretize a continuous model and analyses of this obtained discrete-time model. In our study, we have determined the basic reproduction number of the discrete model according to the work of Micheal A. mikucki (2012) [22] and study the existence and the stability of the diseasefree and endemic equilibrium points of the model. Finally numerical simulations are performed to show the dynamic behavior of SIR model.

\section{Methods and Materials}

A. Materials: In our study we use the parameter values that used Dubey et al. [1] in their continous model. Because mainly the data of a epidemic model is collected in discrete time.Here we give the parameters as $A=7, \delta_{0}=0.02, \delta_{1}=1, \delta_{2}=0.05, a=0.2, b=0.02, \alpha=0.003, \beta=0.002, \gamma=0.5$

B. Method: We convert a continuous model into a discrete model using Euler discretization Scheme. And showed the effect of step size $h$.

\section{Discrete Sir Model}

In this study, we reconsider a SIR epidemic model with nonlinear incidence and treatment rate derived by (Dubey et al. 2015) [1]:

$\frac{d S}{d t}=A-\delta_{0} S-\frac{\alpha S I}{1+\beta S+\gamma I}$

$\frac{d I}{d t}=\frac{\alpha S I}{1+\beta S+\gamma I}-\delta_{0} I-\delta_{1} I-\delta_{2} I-\frac{a I}{1+b I}$

(1)

$\frac{d R}{d t}=\delta_{2} I-\delta_{0} R+\frac{a I}{1+b I}$

Where, $S(0)>0, \quad I(0) \geq 0, \quad R(0) \geq 0$

Here, $S(t), I(t)$ and $R(t)$ represent the number of susceptible, infective and recovered individuals at time $\mathrm{t}$ respectively. The recruitment of susceptible is $\mathrm{A}$ and $\delta_{0}$ be the natural death rate of the population in each class. $\delta_{1}$ is the death of infected individuals due to infection, $\delta_{2}$ is the natural recovery rate of infected individuals due to immunity. In model (1) dubey et al took the incidence rate as BeddingtonDe Angelis type: 


$$
f(S, I)=\frac{\alpha S I}{1+\beta S+\lambda I}
$$

Here $\alpha$ is the transmission rate, $\beta$ is a measure of inhibition effect, such as preventive measure taken by susceptible individuals, and $\gamma$ is measure of inhibition effect such as treatment with respect to inflectives. This nonlinear incidence rate is more reasonable than bilinear incidence rate.

In model (1) we see, the person has recovered once from the disease, that means the person received immunity. The discrete epidemic model is more suitable to describe the spread of diseases since the epidemiological data are usually collected in discrete time units, such as daily, weekly, or monthly. We are interested to derive the discrete version of model (1). Applying Euler Scheme to system (1) leads to discrete systems as follows that shows in Fig 1:

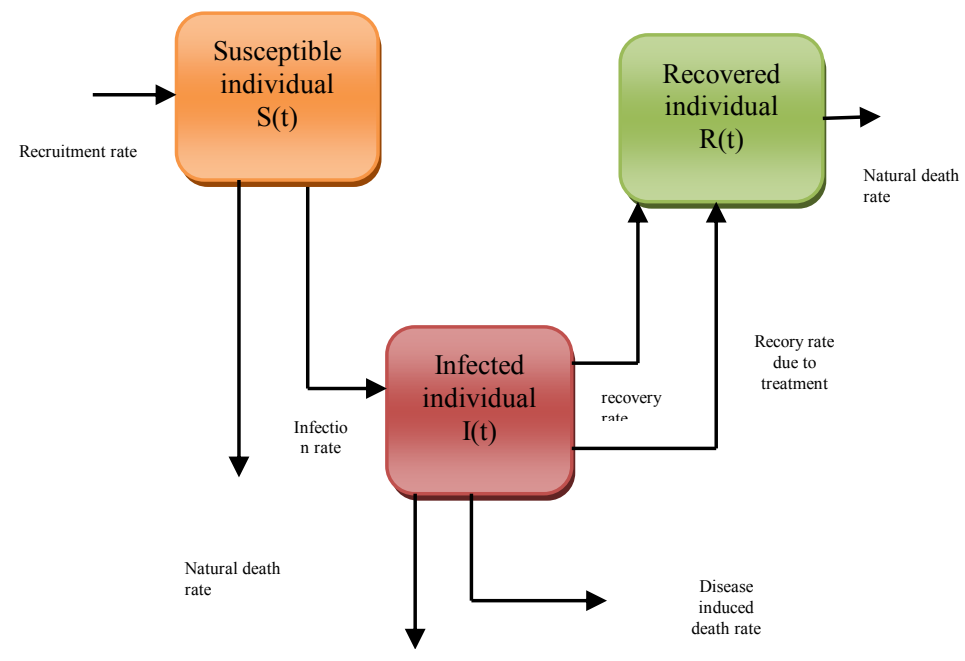

$$
\begin{aligned}
& \text { Natural death } \\
& \text { rate }
\end{aligned}
$$

Fig 1 Flowchart of SIR model

So, the formulated Discrete SIR model is as follows:

$$
\begin{aligned}
& S_{n+1}=S_{n}+h\left\lfloor A-\delta_{0} S_{n}-\frac{\alpha S_{n} I_{n}}{1+\beta S_{n}+\gamma I_{n}}\right\rfloor \\
& I_{n+1}=I_{n}+h\left\lfloor\frac{\alpha S_{n} I_{n}}{1+\beta S_{n}+\gamma I_{n}}-\delta_{0} I_{n}-\delta_{1} I_{n}-\delta_{2} I_{n}-\frac{a I_{n}}{1+b I_{n}}\right\rfloor
\end{aligned}
$$

$$
R_{n+1}=R_{n}+h\left\lfloor\delta_{2} I_{n}-\delta_{0} R_{n}-\delta_{2} I_{n}+\frac{\alpha I_{n}}{1+b I_{n}}\right\rfloor
$$

Where $h>0$ is the step size of integration. Due to the biological interpretation of system (2), only nonnegative solutions are meaningful to be considered.

\section{Analytical Analysis of the Discrete Model 4.1. Positivity and the Boundedness of the Discrete Model Solution:}

Theorem 3.1 All solutions of the model (2) with nonnegative initial value remain non negative for all $t \geq 0$ and are ultimately bounded.

Proof: In our model, we consider $S(t), I(t)$ and $R(t)$ the number of susceptible, infective and recovered individuals at time t respectively. Let, $N_{n}$ be the total number of population at time n. So, $N_{n}=S_{n}+I_{n}+R_{n}$

Adding all equations in model (2) we obtain the equation for the total population on $N_{n}$.

Substituting $S_{n}=N_{n}-I_{n}-R_{n}$ into second equation of model (2), we get,

$$
\begin{aligned}
& N_{n+1}=N_{n}+h\left[A-\delta_{1} I_{n}-\delta_{0} N_{2}\right] \\
& R_{n+1}=R_{n}+h\left[\delta_{2} I_{n}+\delta_{0} R_{n}+\frac{a I_{n}}{1+\delta I_{n}}\right]
\end{aligned}
$$

viously, model (2) and model (3) are equivalent.

Now from $1^{\text {st }}$ equation we get,

$$
\begin{aligned}
& N_{n+1}=N_{n}+h\left[A-\delta_{1} I_{n}-\delta_{0} N_{n}\right] \\
& N_{n+1}<h A+\left(1-\delta_{0} h\right) N_{n} \\
& N_{n+1}<h A+\left(1-\delta_{0} h\right)\left[h A+\left(1-\delta_{0} h\right) N_{n-1}\right. \\
& N_{n+1}<h A+h A\left(1-\delta_{0} h\right)+\left(1-\delta_{0} h\right)^{2} N_{n-1} \\
& N_{n+1}<h A\left[1+\left(1-\delta_{0} h\right)\right]+\left(1-\delta_{0} h\right)^{2} N_{n-1} \\
& N_{n+1}<h A\left[\frac{1-\left(1-\delta_{0} h\right)^{2}}{\delta_{0} h}\right]+\left(1-\delta_{0} h\right)^{2} N_{n-1}
\end{aligned}
$$

Which implies.

$$
N_{n+1}<h A\left[\frac{1-\left(1-\delta_{0} h\right)^{n+1}}{\delta_{0} h}\right]+\left(1-\delta_{0} h\right)^{n+1} N_{0}
$$

So, $N_{n}<h A\left[\frac{1-\left(1-\delta_{0} h\right)^{n}}{\delta_{0} h}\right]+\left(1-\delta_{0} h\right)^{n} N_{0}$

Now, $N_{n} \geq 0$ for $n_{n} \geq 0$ when the initial value $N_{n} \geq 0$

Similar we can show that $I_{n}$ and $R_{n} \geq 0$ for $n_{n} \geq 0$ for initial condition $I_{0} \geq 0$ and $R_{0} \geq 0$ respectively.

Now we will show that, the solutions system is ultimately bounded.

It is clear that the equation $N_{n+1}=N_{n}+h\left[A-\delta_{1} I_{n}-\delta_{0} N_{n}\right]$ has a unique equilibrium $N_{*}=\frac{h A}{\delta_{0}}-\frac{\delta_{1} I^{*}}{\delta_{0}}$ 
i.e $N_{*} \leq \frac{h A}{\delta_{0}}$, which is globally asymptotically stable, that is $\lim _{t \rightarrow \infty} N_{n}=N_{0}$

Therefore, $\lim _{t \rightarrow+\infty} S P\left(S_{n}+I_{n}+R_{n}\right) \leq \frac{h A}{\delta_{0}}$

It follows that the model (2) is bounded in the fesible region,

$\Omega=\left\{(S, I, R): S, I, R \geq 0, S+I+R \leq \frac{h A}{\delta_{0}}\right\}$

Obviously, this region is positively invariant with respect to the model (2) which implies the model (2) is ultimately bounded.

\subsection{Equilibrium points:}

In this study only gets two positive equilibrium points . one is disease free equilibrium point (DFE) and the another is endemic equilibrium point.

For find the equilibrium points of the systems (2)

Set, $S_{n+1}=S_{n}=S^{*}$

$I_{n+1}=I_{n}=I^{*}$

$R_{n+1}=R_{n}=R^{*}$

Then the system (2) reduces to,

$A-\delta_{0} S^{*}-\frac{\alpha S^{*} I^{*}}{1+\beta S^{*}+\gamma I^{*}}=0$

$\frac{\alpha S^{*} I^{*}}{1+\beta S^{*}+\gamma I^{*}}-\left(\delta_{0}+\delta_{1}+\delta_{2}\right) I^{*}-\frac{a I^{*}}{1+b I^{*}}=0$

(4)

$$
\delta_{2} I-\delta_{0} R+\frac{a I^{*}}{1+b I^{*}}=0
$$

\subsection{Disease free equilibrium point (DFE):}

Now we investigate the disease free equilibrium point of the model (2)

At Disease free equilibrium, $I^{*}=R^{*}=0$ and put $S^{*}=S_{0}$

Then the system (3) reduces to,

$A-\delta_{0} S_{0}=0$

$\therefore S_{0}=\frac{A}{\delta_{0}}$

So the disease free- equilibrium point of the system (2) is,

$E_{0}=\left(\frac{A}{\delta_{0}}, 0,0\right)$

\subsection{Basic reproduction number:}

In model (2) the first two equation are independent of third equation. So, for analysis the model we can consider only the first two equations.

$$
\begin{aligned}
& I_{n+1}=I_{n}+h\left\lfloor\frac{\alpha S_{n} I_{n}}{1+\beta \delta_{n}+\gamma I_{n}}-\delta_{3} I_{n}-\frac{a I_{n}}{1+b I_{n}}\right\rfloor \\
& S_{n+1}=S_{n}+h\left\lfloor A-\delta_{0} S_{n}-\frac{\alpha S_{n} I_{n}}{1+\beta \delta_{n}+\gamma I_{n}}\right\rfloor
\end{aligned}
$$

Where, $\delta_{3}=\delta_{0}+\delta_{1}+\delta_{2}$

The Jacobian matrix of the system (5) at DEF is-

$$
J\left(0, \frac{A}{\delta_{0}}\right)=\left(\begin{array}{cc}
1+\frac{h \alpha A}{\delta_{0}+\beta A}-\delta_{3} h-a h & 0 \\
-\frac{h \alpha A}{\delta_{0}+\beta A} & 1+h \delta_{0}
\end{array}\right)
$$

So according to the work of Michael A. Mikucki (2012) [22], we get,

$$
\begin{aligned}
& F+T=\left[\frac{h \alpha A}{\delta_{0}+\beta A}+1-\delta_{3} h-a h\right] \\
& A=\left[-\frac{h \alpha A}{\delta_{0}+\beta A}\right] \text { and } c=\left[1+h \delta_{0}\right]
\end{aligned}
$$

Now we consider,

$$
\begin{aligned}
& F=\left[\frac{h \alpha A}{\delta_{0}+\beta A}\right] \\
& T=\left[1-\delta_{3} h-a h\right]
\end{aligned}
$$

The basic reproduction number of the system (2) will be $p\left(F[I-T]^{-1}\right)$.

Where, $I$ is an $1 \times 1$ identity matrix.

$$
\begin{aligned}
\therefore 1-T & =\left[1-\left(1-\delta_{3} h-a h\right)\right. \\
& =\left[a h+\delta_{3} h\right]
\end{aligned}
$$

Or $[1-T]^{-1}=\left[\frac{1}{a h+\delta_{3} h}\right]$

Now, $F[1-T]^{-1}=\left[\frac{h \alpha A}{\delta_{0}+\beta A}\right] \cdot\left[\frac{1}{a h+\delta_{3} h}\right]$

$$
=\left\lfloor\frac{\alpha A}{\left(\delta_{0}+\beta A\right)\left(\delta_{3}+a\right)}\right\rfloor
$$

$\therefore F[1-T]^{-1}=\left\lfloor\frac{\alpha A}{\left(\delta_{0}+\beta A\right)\left(\delta_{3}+a\right)}\right\rfloor$

$\therefore R_{0}=\frac{\alpha A}{\left(\delta_{0}+\beta A\right)\left(\delta_{3}+a\right)}$

Which is the same of the continuous model (1).

\subsection{Endemic equilibrium point:}

In this section, we observe the endemic equilibrium points of the model: 
We find the endemic equilibrium point by solving systems (4)

From third equation of the system (4) we get,

$$
\begin{aligned}
& \delta_{2} I^{*}-\delta_{2} R^{*}+\frac{a I^{*}}{1+b I^{*}}=0 \\
& R^{*}=\frac{\delta_{2} I^{*}+b \delta_{2} I^{* 2}+a I^{*}}{\delta_{0}\left(1+b I^{*}\right)} \\
& R^{*}=\frac{\left(a+\delta_{2}\right) I^{*}+b \delta_{2} I^{* 2}}{\delta_{0}\left(1+b I^{*}\right)}
\end{aligned}
$$

(6)

From $2^{\text {nd }}$ equation of system (4) we obtain,

$$
\begin{aligned}
& \frac{\alpha S^{*} I^{*}}{1+\beta S^{*}+\gamma I^{*}}-\left(\delta_{0}+\delta_{1}+\delta_{2}\right) I^{*}-\frac{a I^{*}}{1+b I^{*}}=0 \\
& \frac{\alpha S^{*} I^{*}}{1+\beta S^{*}+\gamma I^{*}}-\delta_{3} I^{*}-\frac{a I^{*}}{1+b I^{*}}=0 \\
& \frac{\alpha S^{*} I^{*}-\delta_{3} I^{*}-\beta \delta_{3} S^{*} I^{*}-\gamma \delta_{3} I^{*^{2}}}{1+\beta S^{*}+\gamma I^{*}}-\frac{a I^{*}}{1+b I^{*}}=0 \\
& \frac{\left(\alpha S^{*} I^{*}-\delta_{3} I^{*}-\beta \delta_{3} S^{*} I^{*}-\gamma \delta_{3} I^{*^{2}}\right)\left(1+b I^{*}\right)-\alpha I^{*}\left(1+\beta S^{*}+\gamma \delta\right)}{\left(1+\beta S^{*}+\gamma I^{*}\right)\left(1+b I^{*}\right)}=0
\end{aligned}
$$

$\left(\alpha-\beta \delta_{3}\right) S^{*} I^{*}+b\left(\alpha-\beta \delta_{3}\right) S^{*} I^{*^{2}}-\delta_{3} I^{*}-b \delta_{3} I^{* 2}-\gamma \delta_{3} I^{* 2}-b \gamma \delta_{3} I^{3^{3}}-a I^{*}-a \beta S^{*} I^{*}-a \gamma I^{*^{2}}=0$

$\left[\left(\alpha-\beta \delta_{3}-a \beta\right) I^{*}+b\left(\alpha-\beta \delta_{3}\right) I^{* 2}\right] S^{*}=\delta_{3} I^{*^{2}}+b \delta_{3} I^{\pi^{2}}+\gamma \delta_{3} I^{*^{2}}+b \gamma \delta_{3} I^{*^{2}}+a I^{*}+a \gamma I^{*}$

$\left[\left(\alpha-\beta \delta_{3}-a \beta\right) I^{*}+b\left(\alpha-\beta \delta_{3}\right) I^{* 2}\right] S^{*}=\left(\delta_{3}+a\right)+\left(b \delta_{3}+\gamma \delta_{3}+a \gamma\right) I^{*}+b \gamma \delta_{3} I^{*^{2}}$

$S^{*}=\frac{\left(a+\delta_{3}+b \delta_{3} I^{*}\right)\left(1+\gamma I^{*}\right)}{\left(\alpha-\beta \delta_{3}-a \beta\right)+b\left(\alpha-\beta \delta_{3}\right) I^{*}}$

$S^{*}$ is positive of $\alpha>\left(S_{3}+a\right) \beta$

(8)

Now form the first equation of systems (4) we get,

$$
\begin{aligned}
& A-\delta_{0} S^{*}-\frac{\alpha S^{*} I^{*}}{1+\beta S^{*}+\gamma I^{*}}=0 \\
& A\left(1+\beta S^{*}+\gamma I^{*}\right)-S_{0} S^{*}\left(1+\beta S^{*}+\gamma I^{*}\right) \alpha S^{*} I^{*}=0 \\
& S_{0} \beta S^{* 2}+\left(S_{0}-A \beta+\left(\delta_{0} \gamma+\alpha\right) I^{*}\right) S^{*}-A\left(1+\gamma I^{*}\right)=0
\end{aligned}
$$

(9)

Substituting the value of $S^{*}$ from equation (7) into equation (9), we get,

$$
A_{1} I^{* 3}+A_{2} I^{* 2}+A_{3} I^{*}+A_{4}=0
$$

Where, $A_{1}=\delta_{0} \beta \gamma \delta_{3}{ }^{2} b^{2}+\delta_{3} b^{2} p l$

$\left.A_{2}=\delta_{0} \beta \gamma \delta_{3}^{2} b^{2}+2 \delta_{0} \delta_{3}\left(\delta_{3}+a\right) \beta \lambda b+b \gamma_{3} q l+b p\left(\delta_{3}+a\right) l+\delta_{3} b m-A b p\right)$.

$A_{3}=2 \delta_{0} \delta_{3}\left(\delta_{3}+a\right) \beta b+\delta_{0} \beta \gamma\left(\delta_{3}+a\right)^{2}+\left(\delta_{3}+a\right) b m p+q\left(\left(\delta_{3}+a\right) l+\delta_{3} b m-2 A b\right.$

$A_{4}=\delta_{0} \beta\left(\delta_{3}+a\right)^{2}+\left(\delta_{3}+a\right) m q-A q^{2}$

And $p=\left(\alpha-\delta_{3} \beta\right), q=\left(a-\delta_{3} \beta-a \beta\right), l=\left(\delta_{0} \lambda+\alpha\right)$

$m=\left(\delta_{0}-A \beta\right)$

It may be noted that $p, q>0$ under condition (8), now using Discartes' rule of sign, the cubic equation (10) has unique positive real root $I^{*}$ if any one of the following holds:

(i) $A_{2}>0, A_{3}>0$ And $A_{4}<0$,

(ii) $A_{2}>0, A_{3}<0$ and $A_{4}<0$,

(iii) $A_{2}>0, A_{3}<0$ and $A_{4}<0$,

We consider first two cases form which we have the following inequalities.

$\left(\delta_{3}+a\right) l+\delta_{0} \delta_{3} b>A \alpha b$

And $R_{0}>1$

After the value of $I^{*}$, we can find the value of $S^{*}$ from equation (7). This implies that there exists a unique endemic equilibrium $E^{*}\left(S^{*}, I^{*}, R^{*}\right)$, if the inequalities (8), (11), (12) are satisfied.

And the equilibrium points of this discrete systems will be the same of the continuous systems.

\subsection{Stability analysis:}

We want to investigate the stability of each fixed points. We note that, the first two equations of the model (2) is independent of $R_{n}$ and therefore we can consider only these two equations for the analysis.

We denote $\psi(S, I)=\frac{\alpha S I}{1+\beta S+\gamma I}$

And $\omega(I)=\frac{\alpha I}{1+\beta I}$

Now the Jacobian matrix of the first two equations of model (2) at a fixed point $\left(S^{e}, I^{e}\right)$ is given by,

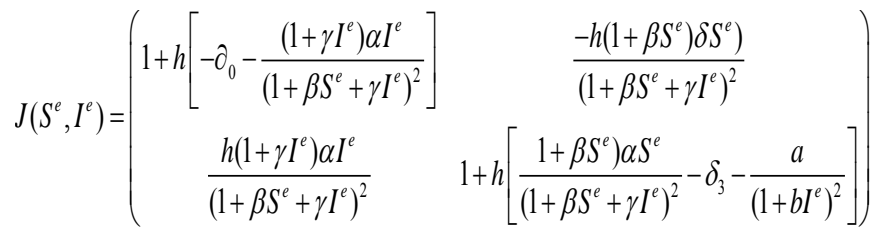

To explore the stability of the fixed points that means at the equilibrium points of model (2), we recall Lemma see. E.g (Das et al, 2011) [23] , (Elsansuny et al. 2012) [24], (Hc, teny and Jiang, 2012) [25]. 


\section{Lemma 1:}

Let, $Q(\lambda)=\lambda^{2}-P_{1} \lambda+P_{2}$ and $Q(1)>0$.

Suppose $\lambda_{1}, \lambda_{2}$ are two roots of $Q(\lambda)=0$. Then

1. $\left|\lambda_{1}\right|<1$ and $\left|\lambda_{2}\right|<1$ if and only if $Q(-1)>0$ and

$$
Q(0)<-1
$$

2. $\left|\lambda_{1}\right|<1$ and $\left|\lambda_{2}\right|>1$ if and only if $Q(-1)<0$;

3. $\left|\lambda_{1}\right|>1$ and $\left|\lambda_{2}\right|>1$ if and only if $Q(-1)>0$ and $Q(0)>1$

4. $\lambda_{1}=-1$ and $\lambda_{2} \neq 1$ if and only if $Q(-1)=0$ and $p_{1} \neq 0,2$;

5. $\lambda_{1}, \lambda_{2}$ are the complex and $\left|\lambda_{1}\right|=1,\left|\lambda_{2}\right|=1$ if and only if $p_{1}^{2}-4 p_{2}<0$ and $Q(0)=1$

A fixed point $\left(S^{e}, I^{e}\right)$ is called a sink if $\left|\lambda_{1}\right|<1$ and $\left|\lambda_{2}\right|<1$, $\left(S^{e}, I^{e}\right)$ is called a saddle. $\left|\lambda_{1}\right|<1$ and $\left|\lambda_{2}\right|>1,\left(S^{e}, I^{e}\right)$ is called a non-hyperbolic if $\left|\lambda_{1}\right|=1$ or $\left|\lambda_{2}\right|=1$.

\section{Stability at Disease free Equilibrium:}

Here we investigate the stability of $E_{0}$

Theorem 3.2: we attain the following assumption.

(i) $E_{0}$ is a sink when $0<\delta_{0} h<2$ and

$$
0<\left(\delta_{0} h+a h\right)\left(1-R_{0}\right)<2
$$

(ii) $E_{0}$ is a stable when, $0<\delta_{0} h<2$ and

$$
\left(\delta_{0} h+a h\right)\left(1-R_{0}\right)>2
$$

(iii) $E_{0} \quad$ is a source, when, $\delta_{0} h>2$ and

$$
\left(\delta_{0} h+a h\right)\left(1-R_{0}\right)>2
$$

(iv) $E_{0}$ is a non-hyperbolic when $\delta_{0}=0$ and $R_{0}=1$

\section{Proof:}

The Jacobian matrix at the disease free equilibrium point $\left(\frac{A}{\delta_{0}}, 0\right)$ is,

$J\left(\frac{A}{\delta_{0}}, 0\right)=\left(\begin{array}{cc}1-\delta_{0} h & \frac{-h \alpha A}{\left(\delta_{0}+\beta A\right)} \\ 0 & 1+\frac{h \alpha A}{\delta_{0}+\beta A}-\delta_{3} h-a h\end{array}\right)$
The Eigen values of Jacobian matrix (ii) are $\lambda_{1}=1-\delta_{0} h$ and

$$
\begin{aligned}
& \lambda_{2}=1+\frac{h \alpha A}{\delta_{0}+\beta A}-\delta_{3} h-a h \\
& =\frac{h \alpha A}{\delta_{0}+\beta A}-\left(\delta_{3} h+a h-1\right) \\
& =1+\frac{h \alpha A}{\delta_{0}+\beta A}-\left(\delta_{3} h+a h\right) \\
& =1-\left(\delta_{3} h+a h\right)+\frac{h \alpha A}{\delta_{0}+\beta A} \\
& =1-\left(\delta_{3} h+a h\right)\left[1-\frac{h \alpha A}{\left(\delta_{0}+\beta A\right)\left(\delta_{3} h+a h\right)}\right] \\
& =1-\left(\delta_{3} h+a h\right)\left[1-\frac{\alpha A}{\left(\delta_{0}+\beta A\right)\left(\delta_{3}+a\right)}\right] \\
& =1-\left(\delta_{3} h+a h\right)\left[1-R_{0}\right]
\end{aligned}
$$

If $R_{0}<1$ then $\lambda_{2} \leq 1$ so we obtain;

(i) $E_{0}$ will be a sink if and only if $\left|\lambda_{1}\right|<1$ and $\left|\lambda_{2}\right|<1$

$$
\begin{aligned}
& \therefore\left|1-\delta_{0} h\right|<1 \\
& \Rightarrow-1<\left|1-\delta_{0} h\right|<1 \\
& \Rightarrow-1-1<\left|1-\delta_{0} h-1\right|<1-1 \\
& \Rightarrow-2<\left|-\delta_{0} h\right|<0 \\
& \Rightarrow 0<\left|\delta_{0} h\right|<2
\end{aligned}
$$

And,

$$
\begin{aligned}
& 1-\left(\delta_{3} h+a h\right)\left[1-R_{0}\right]<1 \\
& \Rightarrow-1<1-\left(\delta_{3} h+a h\right)\left[1-R_{0}\right]<1 \\
& \Rightarrow-1-1<1-\left(\delta_{3} h+a h\right)\left[1-R_{0}\right]-1<1-1 \\
& \Rightarrow-2<-\left(\delta_{3} h+a h\right)\left[1-R_{0}\right]<0 \\
& \Rightarrow 0<-\left(\delta_{3} h+a h\right)\left[1-R_{0}\right]<2
\end{aligned}
$$

(ii) $E_{0}$ will be a saddle if and only if

$$
\left|\lambda_{1}\right|<1 \text { and }\left|\lambda_{2}\right|>1
$$

Now,

$$
\begin{aligned}
& \therefore\left|1-\delta_{0} h\right|<1 \\
& \Rightarrow-1<\left|1-\delta_{0} h\right|<1 \\
& \Rightarrow 0<\left|\delta_{0} h\right|<2
\end{aligned}
$$

And 


$$
\begin{aligned}
& \left|\lambda_{2}\right|>1 \\
& \text { i.e, }\left(\delta_{3} h+a h\right)\left[1-R_{0}\right]>2
\end{aligned}
$$

(iii) $E_{0}$ will be a source, if $\left|\lambda_{1}\right|>1$ and $\left|\lambda_{2}\right|>2$

Which implies,

$\delta_{0} h>2$ and $\left(\delta_{3} h+a h\right)\left[1-R_{0}\right]>2$

iv) $E_{0}$ is a non-hyperbolic

$$
\text { when }\left|\lambda_{1}\right|=1 \text { or }\left|\lambda_{2}\right|=1
$$

i.e,

$$
\begin{aligned}
& 1-\delta_{0} h=1 \\
& \Rightarrow-\delta_{0} h=0 \\
& \therefore \delta_{0} h=0 \\
& \delta_{0}=0
\end{aligned}
$$

We can say when basic reproduction number is 1 , then $E_{0}$ is a non-hyperbolic.

\section{Stability analysis at endemic Equilibrium:}

Now we can observe the stability of endemic equilibrium $E^{*}$.

The Jacobian matrix at the endemic equilibrium is,

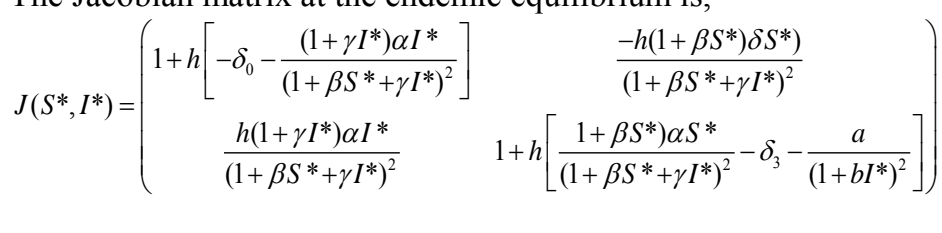

The characteristic equation of this Jacobian matrix is

$$
Q(\lambda)=\lambda^{2}+p_{1} \lambda+p_{2}
$$

Where $p_{1}=T_{r}(J)$ and $p_{2}=x t(J)$

Now consider,

$$
\begin{aligned}
& \psi\left(S^{*}, I^{*}\right)=\frac{\left(1+\gamma I^{*}\right) \alpha I^{*}}{\left(1+\beta S^{*}+\gamma I^{*}\right)^{2}} \\
& \phi\left(S^{*}, I^{*}\right)=\frac{\left.1+\beta S^{*}\right) \alpha S^{*}}{\left(1+\beta S^{*}+\gamma I^{*}\right)^{2}} \\
& f\left(I^{*}\right)=\frac{a}{\left(1+b I^{*}\right)^{2}}
\end{aligned}
$$

$$
\therefore J\left(S^{*}, I^{*}\right)=\left(\begin{array}{cc}
1+h\left(-\delta_{0}+\psi\left(S^{*}, I^{*}\right)\right. & -h \phi\left(S^{*}, I^{*}\right) \\
h \psi\left(S^{*}, I^{*}\right) & 1+h\left[\phi\left(S^{*}, I^{*}\right)-\delta_{3}-\right]
\end{array}\right)
$$

$\therefore P_{1}=2+h\left[-\delta_{0}+\psi\left(S^{*}, I^{*}\right)+\phi\left(S^{*}, I^{*}\right)-\delta_{3}-f\left(I^{*}\right)\right]$

And

$P_{2}=1+h\left[-\delta_{0}+\psi\left(S^{*}, I^{*}\right)\right]+\left(1+h\left(\phi\left(S^{*}, I^{*}\right)-\delta_{3}-f\left(I^{*}\right)+h^{2} \phi\left(S^{*}, I^{*}\right) \psi\left(S^{*}, I^{*}\right)\right.\right.$

$=1+h\left[-\delta_{0}+\psi\left(S^{*}, I^{*}\right)\right]+\phi\left(S^{*}, I^{*}\right)-\delta_{3}-f\left(I^{*}\right)+h^{2}\left[-\delta_{0}+\psi\left(S^{*}, I^{*}\right)\right]\left(\phi\left(S^{*}, I^{*}\right)-\delta_{3}-f\left(I^{*}\right)\right)+\phi\left(S^{*}, I^{*}\right) \psi\left(S^{*}, I^{*}\right)$

Lemma 2: If $R_{0}>1$, then $R(1)>0$.

Proof:

$Q(1)=h^{2}\left[\left(\phi\left(S^{*}, I^{*}\right) \psi\left(S^{*}, I^{*}\right)\right)+\left(\delta_{0}+\psi\left(S^{*}, I^{*}\right)\right)\left(\phi\left(S^{*}, I^{*}\right)-\delta_{3}-f\left(I^{*}\right)\right)\right.$

Now at $P_{0}>1$,

Then $S^{*}>0$ and $I^{*}>0$

Then $\phi\left(S^{*}, I^{*}\right)>0$ and $\psi\left(S^{*}, I^{*}\right)>0$

Since all the parameters are non-negative, it is clear that $Q(0)>1$,

Lemma 3: If $R_{0}>1$, then $Q(0)<1$, is equivalent to $h<h^{*}$ where

$h^{*}=\frac{\delta_{0}+\psi\left(S^{*}, I^{*}\right)-\phi\left(S^{*}, I^{*}\right)+\delta_{3}+f\left(I^{*}\right)}{\left(\psi\left(S^{*}, I^{*}\right)-\delta_{0}\right)\left(\phi\left(S^{*}, I^{*}\right)-\delta_{3}-f\left(I^{*}\right)+\phi\left(S^{*}, I^{*}\right) \psi\left(S^{*}, I^{*}\right)\right.}$

Proof: We note that $R_{0}>1$, implies $\psi\left(S^{*}, I^{*}\right)>0$, $f\left(I_{0}\right)>0$ and $\phi\left(S^{*}, I^{*}\right)>0$

Clearly $Q(0)=p_{2}<1$ is equivalent to

$$
1+h\left[-\delta_{0}+\psi\left(S^{*}, I^{*}\right)\right]+\phi\left(S^{*}, I^{*}\right)
$$

$-\delta_{3}-f\left(I^{*}\right)+h^{2}\left[-\delta_{0}+\psi\left(S^{*}, I^{*}\right)\right]$

$\left(\phi\left(S^{*}, I^{*}\right)-\delta_{3}-f\left(I^{*}\right)\right)+\phi\left(S^{*}, I^{*}\right)$

$\psi\left(S^{*}, I^{*}\right)<1$

$\Rightarrow h<\frac{\delta_{0}+\psi\left(S^{*}, I^{*}\right)-\phi\left(S^{*}, I^{*}\right)+\delta_{3}+f\left(I^{*}\right)}{\left(\psi\left(S^{*}, I^{*}\right)-\delta_{0}\right)\left(\phi\left(S^{*}, I^{*}\right)-\delta_{3}-f\left(I^{*}\right)+\phi\left(S^{*}, I^{*}\right) \psi\left(S^{*}, I^{*}\right)\right.}=h^{*}$

From Lemma 1, 2 and 3 we have the following theorem

Theorem 3.3: If $R_{0}>1$, then we have,

1. $E^{*}$ is a sink if then following conditions holds $h<h^{*}$

2. $E^{*}$ is a saddle if $h_{1}^{*}<h<h_{2}{ }^{*}$

3. $E^{*}$ is a source if $h>h^{*}$

4. $E^{*}$ is a non-hyperbolic if $h=h^{*}$

\section{Numerical Analysis of the Discrete Model}

In this section we present various numerical results using the Euler scheme .To give some numerical evidence for the 
qualitative dynamic behavior of the model (2), we will display some diagrams to illustrate the analytical results and explore a new dynamics behavior as the parameters change. In particular, we present numerical simulation results for model (2) by using Matlab programming language in Matlab 2018b. Since in eqidemic model the data is collected in discrete time. So, we choose some hypothetical data used in the work of Dubey et al. 2015 [1] . Our main goal is to illustrate the result by numerical simulations considered from a qualitative, rather than a quantitative point of view. Along with the verification of our analytical observations, these numerical results are very much important from the practical point of view. The numerical result shows different behavior of our dynamical model. To develop our numercal analysis we follow the work of several researchers in [26-28].

For

$A=7, \delta_{0}=0.02, \delta_{1}=1, \delta_{2}=0.05, a=0.2, b=0.02, \alpha=0.003, \beta=0.002, \gamma=0.5$ at the initial position $(S, I, R)=(245,45,1)$

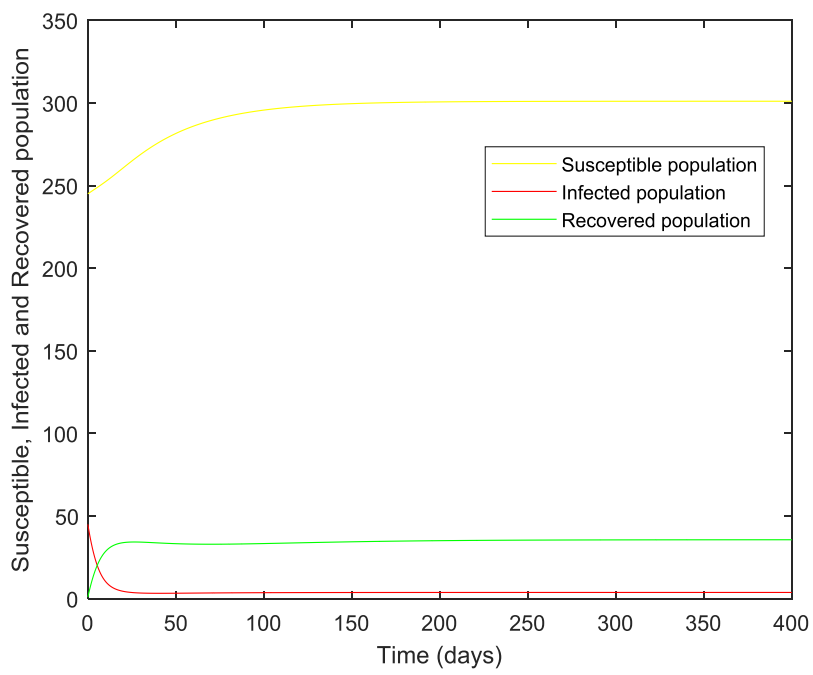

(a) $\mathrm{h}=0.01$

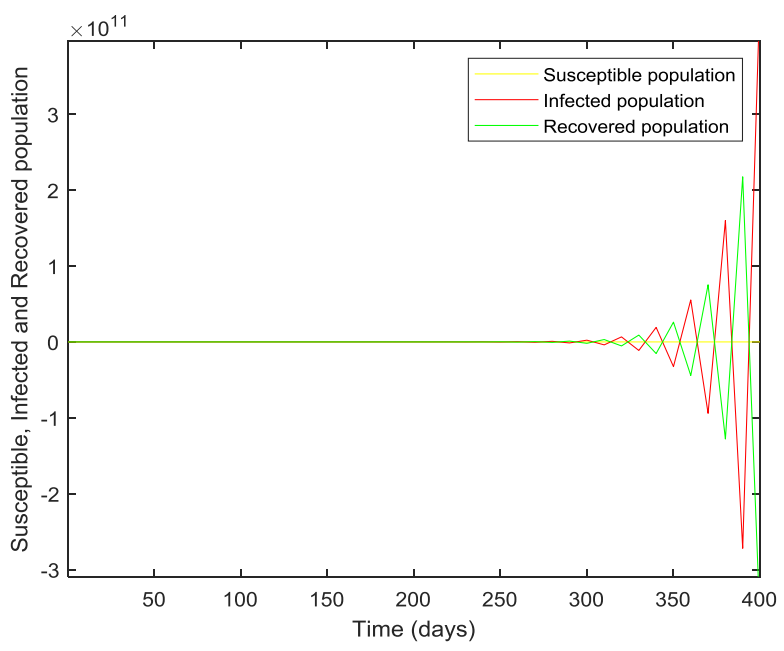

(b) $\mathrm{h}=10$

Fig 2 Susceptible, Infected and Recovered population vs time when treatment ontion is present

In Fig 2, we see that the number of the infected individuals $I(t)$ decreases with time, and these individuals once recovered became immunized. That means they will not get reinfected. Besides, the susceptible individuals $\mathrm{S}(\mathrm{t})$ increases. The decrease of infected individual and the increase of susceptible individuals occur due to treatments.

Case 1: We choose the parameter values

$A=7, \delta_{0}=2, \delta_{1}=1, \delta_{2}=2, a=1, b=0.02, \alpha=1, \beta=1, \gamma=0.1$ at the initial position

$$
(S, I, R)=(245,45,1)
$$
. Here $R_{0}<1$, the average number of a new infection by an infected individual is less than one. So, the equilibrium point $E_{0}$ is asymptotically stable.

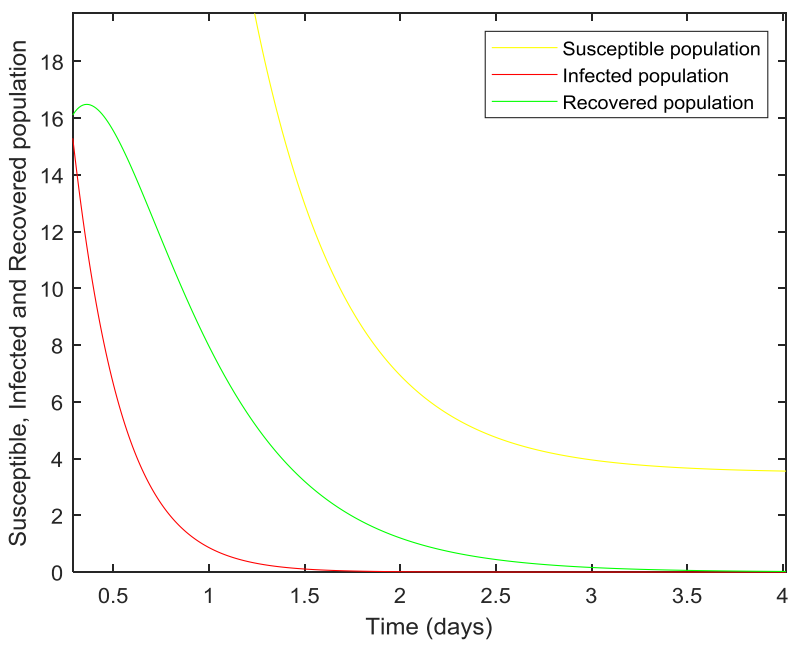

(a) $\mathrm{h}=0.01$ 


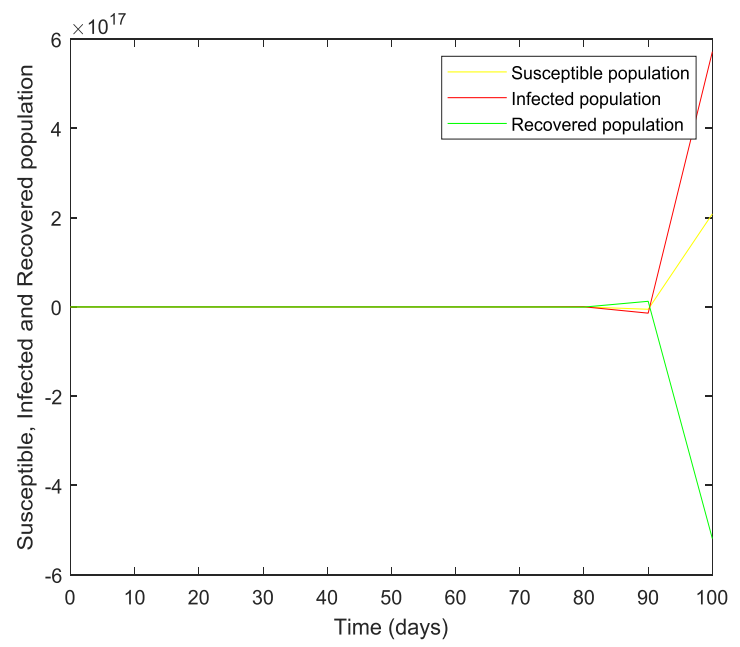

(b) $\mathrm{h}=10$

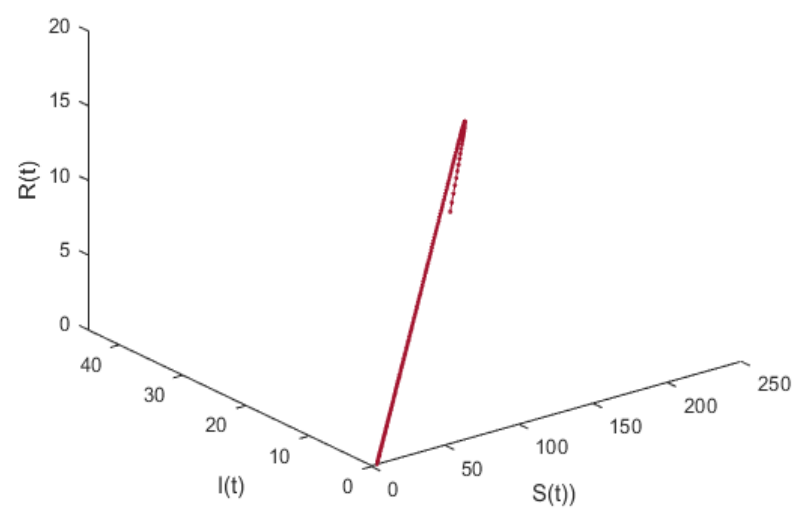

(c) $\mathrm{h}=0.01$

Fig 3 Susceptible, Infected and Recovered population vs time and Phase diagram for the model (2) with $R_{0}<1$

Case 2: We take the parameter values

$A=7, \delta_{0}=2, \delta_{1}=1, \delta_{2}=1, a=1, b=0.02, \alpha=1, \beta=1, \gamma=0.1$ at the initial position $(S, I, R)=(245,45,1)$ . Here $R_{0}=1$, the average number of a new infection by an infected individual is equal to one. So, the disease in these case is constant.

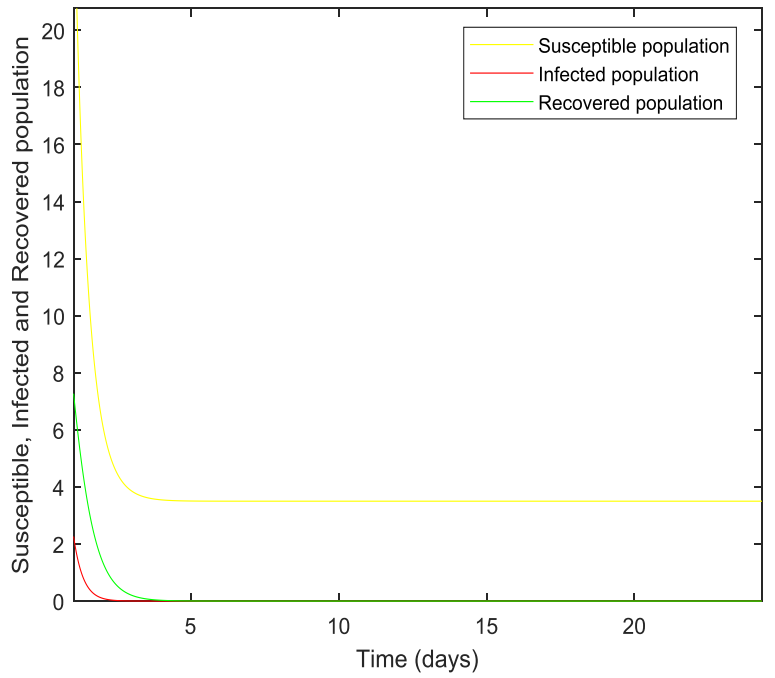

(a)

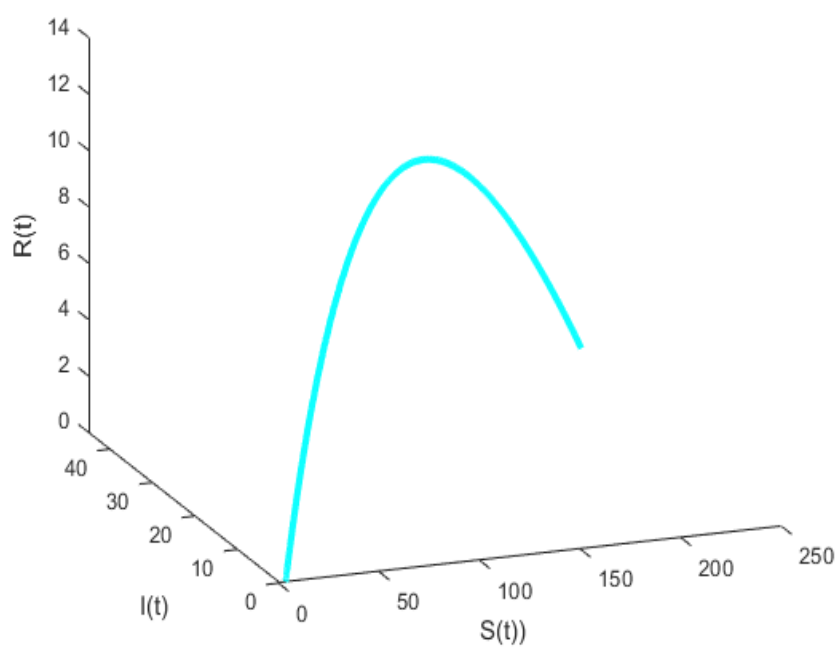

(b)

Fig 4 Susceptible, Infected and Recovered population vs time and Phase diagram for the model (2) with $R_{0}=1$

Case 3: We choose the parameter values $A=7, \delta_{0}=0.002, \delta_{1}=0.005, \delta_{2}=0.01, a=2, b=0.02, \alpha=0.19, \beta=0.02, \gamma=0.005$ at the initial position $R_{0}>1$, the average number of a new infection by an infected individual is more than one. So, the equilibrium point $E^{*}$ is asymptotically stable. 


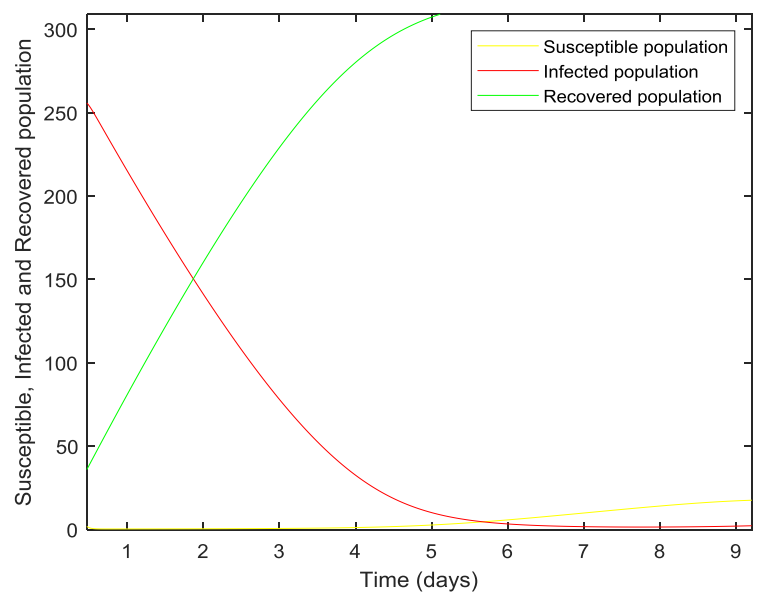

(a) $\mathrm{h}=0.01$

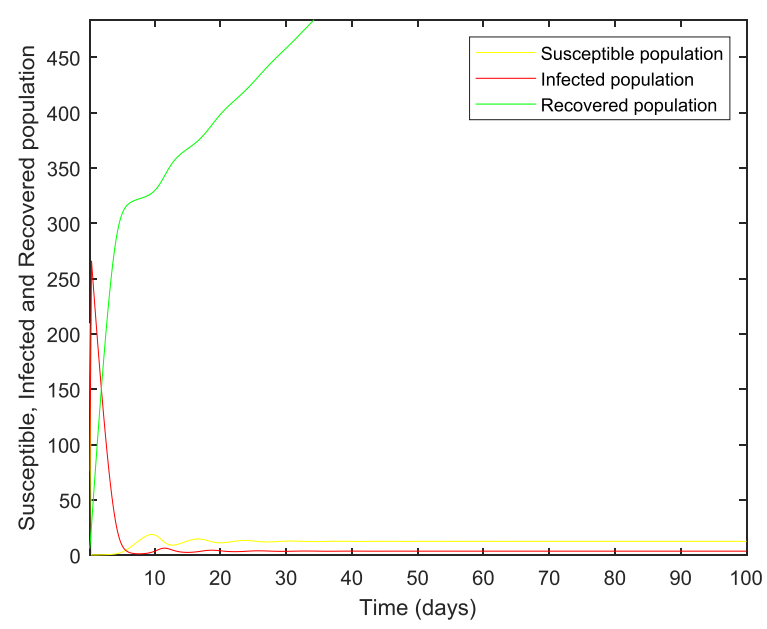

(b) $\mathrm{h}=0.1$

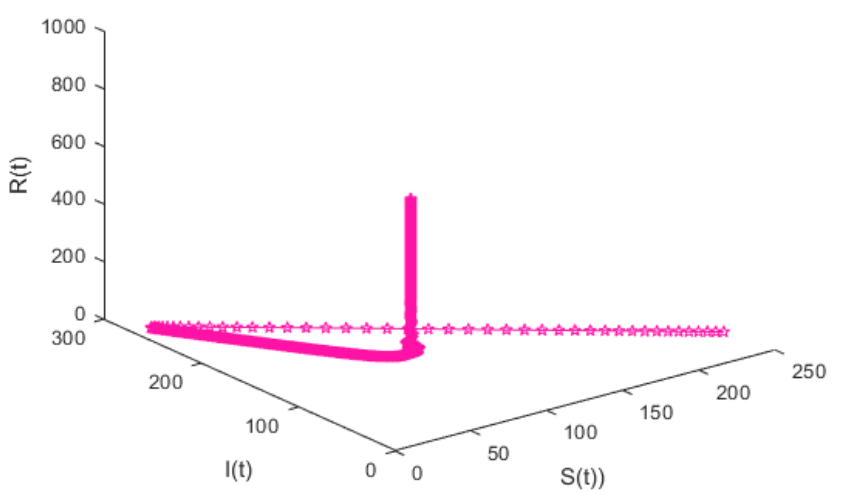

(c) $\mathrm{h}=0.01$

Fig 5 Susceptible, Infected and Recovered population vs time and Phase diagram for the model (2) with $R_{0}>1$

In Fig 6(a) and 6(c), we see the effect of $\alpha$ (incidence rate) on $\mathrm{S}(\mathrm{t})$ and $\mathrm{I}(\mathrm{t})$ individuals (respectively) for given parameters $A=7, \delta_{0}=0.02, \delta_{1}=1, \delta_{2}=0.05, a=0.2, b=0.02, \alpha=0.003, \beta=0.002, \gamma=0.5$ when $\mathrm{h}=0.01$ at the initial value $(S, I, R)=(245,45,1)$. Fig 6(a) shows when $\alpha$ increases, the susceptible individuals $\mathrm{S}(\mathrm{t})$ sharply decline initially and at $\alpha=0.006 \mathrm{~S}(\mathrm{t})$ decreases slowly. Then it harmony to its equilibrium points. Fig 6(c), gives more people will be infected when the incidence rate is larger. And less people will be infected when the incidence rate is lower. For a larger incidence rate, the number of infected individuals increases at the initial position, then decreases and finally sits down at its steady state. Due to immunity and the treatments this decrease occurs. But when $\mathrm{h}=10$, then the effect of $\alpha$ on susceptible cell and infected cell cannot understand clearly.

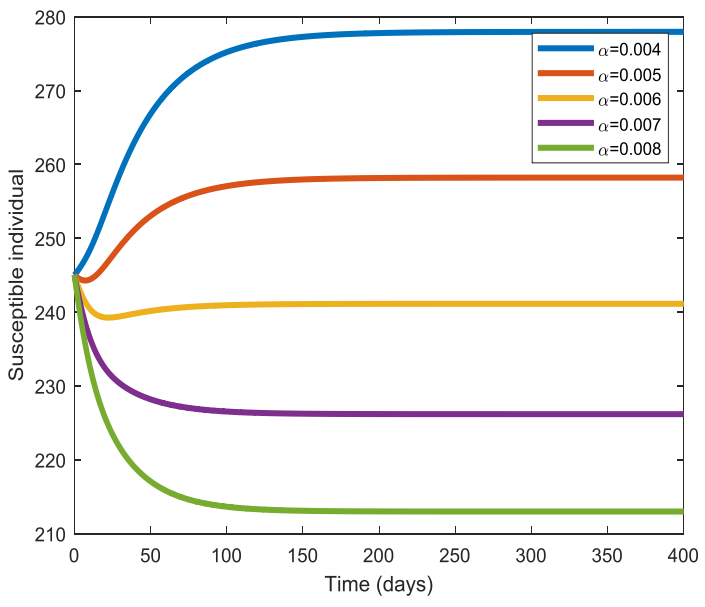

(a) $\mathrm{h}=0.01$ 


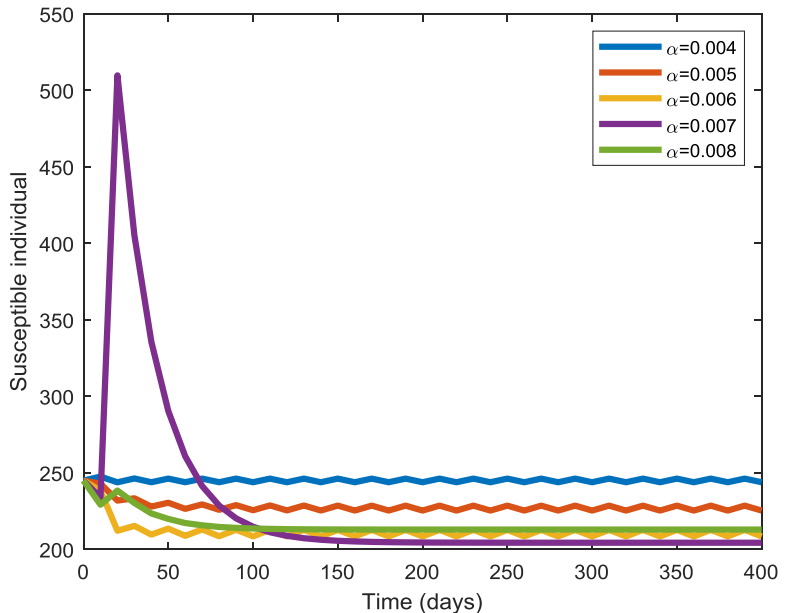

(b) $\mathrm{h}=10$

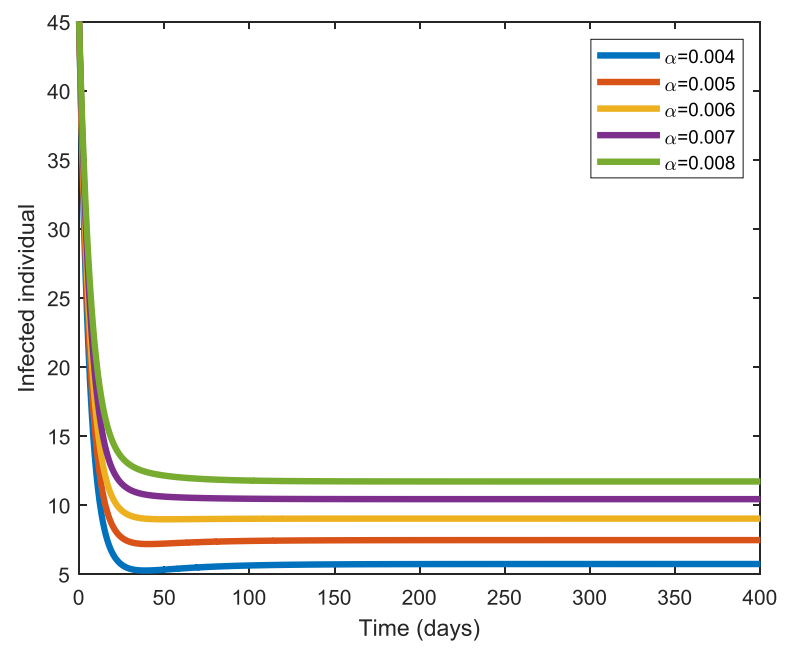

(c) $\mathrm{h}=0.01$

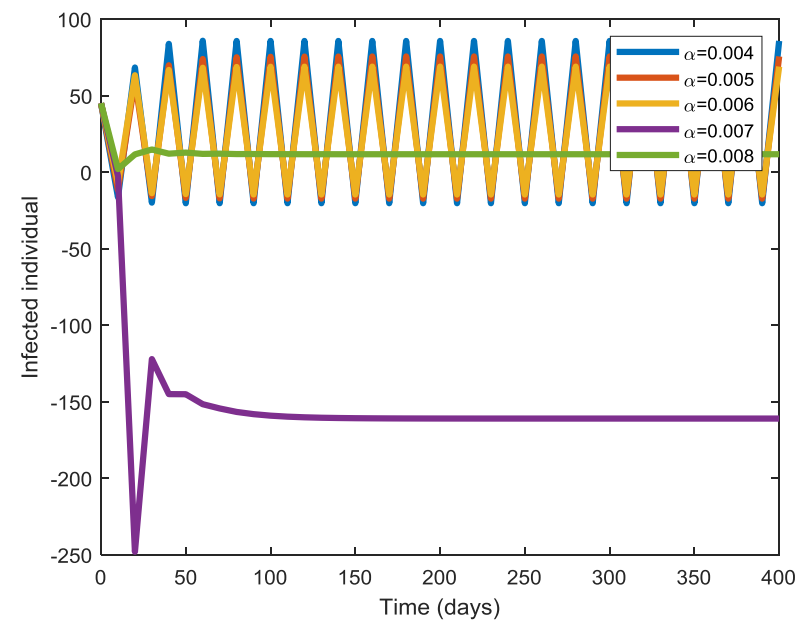

(d) $\mathrm{h}=10$

Fig 6 Effect of $\alpha$ on Susceptible and and infected individuals

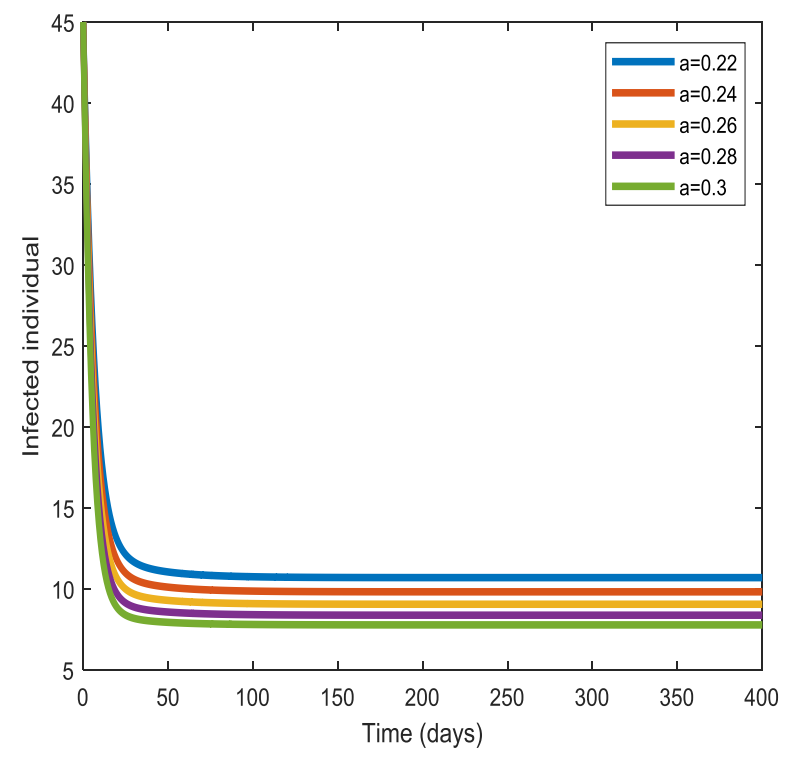

(a) $\mathrm{h}=0.01$ 


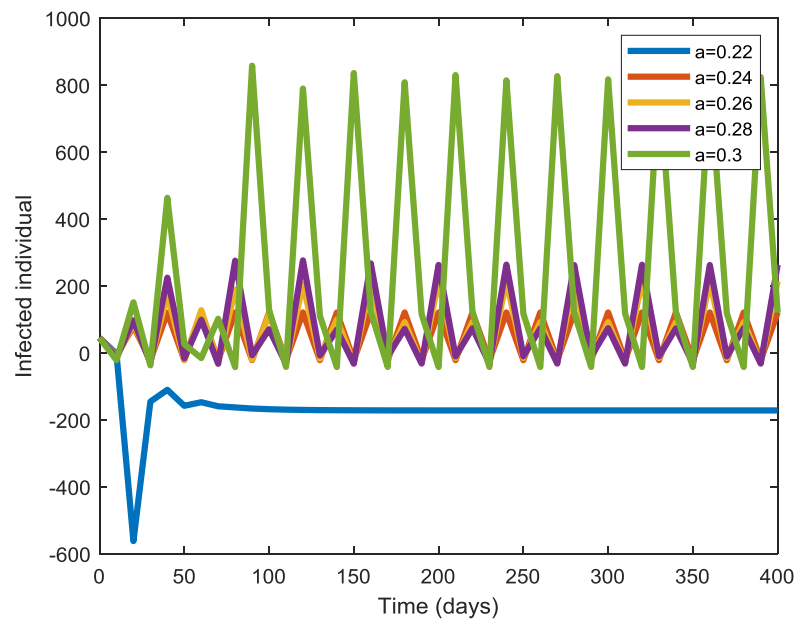

(b) $\mathrm{h}=10$

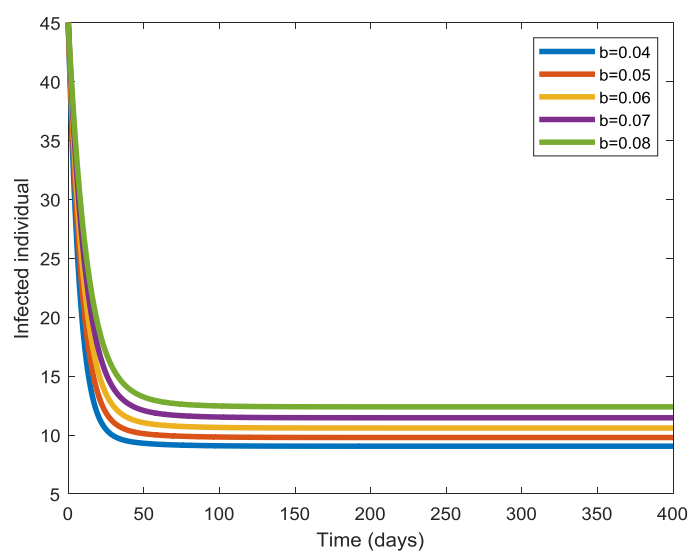

(c) $\mathrm{h}=0.01$

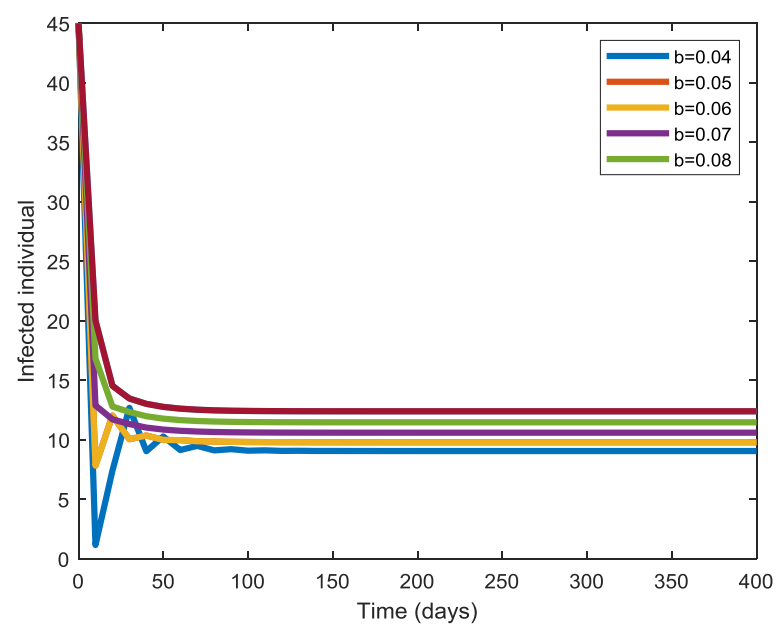

(d) $\mathrm{h}=10$
Now we observe the effect of treatment rate ' $a$ ' and limitation to treatment rate ' $b$ ' on infected population in Fig 7(a) and 7(c). Fig 7(a) narrates infected individuals decreases when treatment rate increases and it sits down at its steady state, but the disease is not getting totally uprooted as it will insist at a much lower level. Fig 7(c) describes infected individuals increases when $b$ increases which is because of limited availability of resources in community when $h=0.01$. But when $h=10$ then the figure more bifurcating than the continuous model.

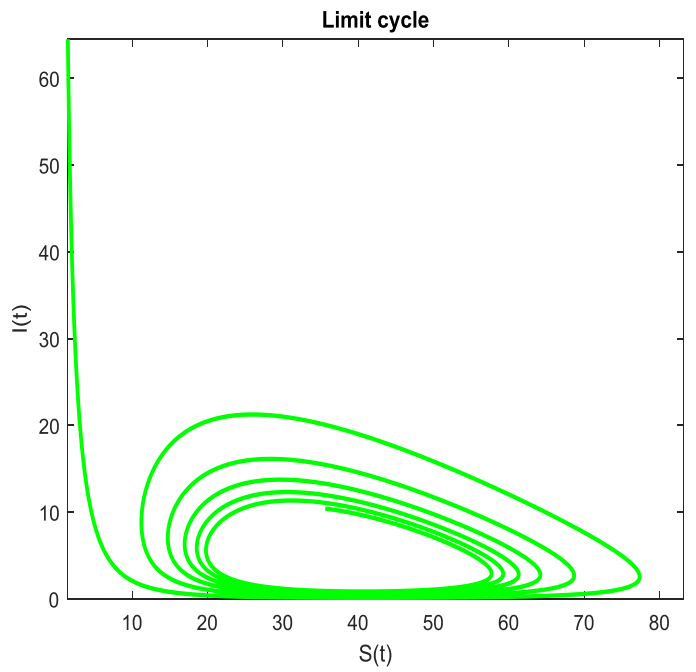

(a) $\mathrm{h}=0.01$

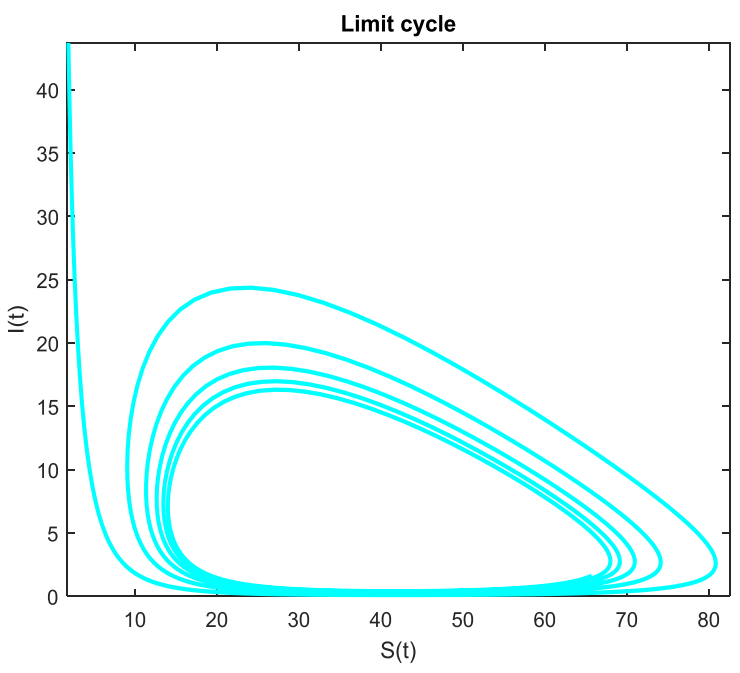

(b) $\mathrm{h}=0.1$

Fig 7 Effect of a and b on Infected individuals 


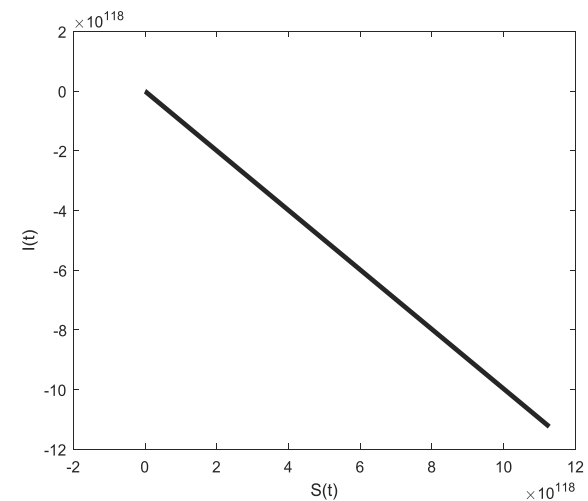

(c) $\mathrm{h}=0.5$

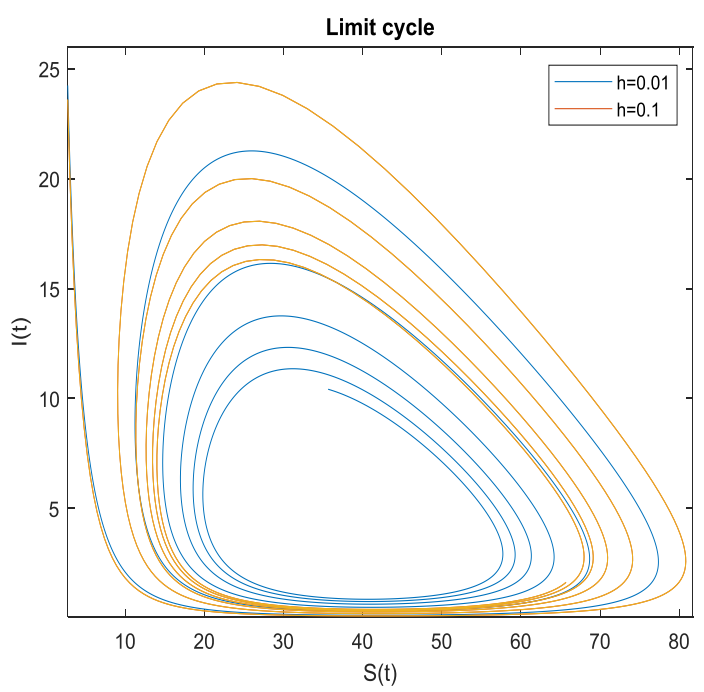

(d) Combined limit cycle

Fig 8 S-I plot for $\alpha=0.08863$

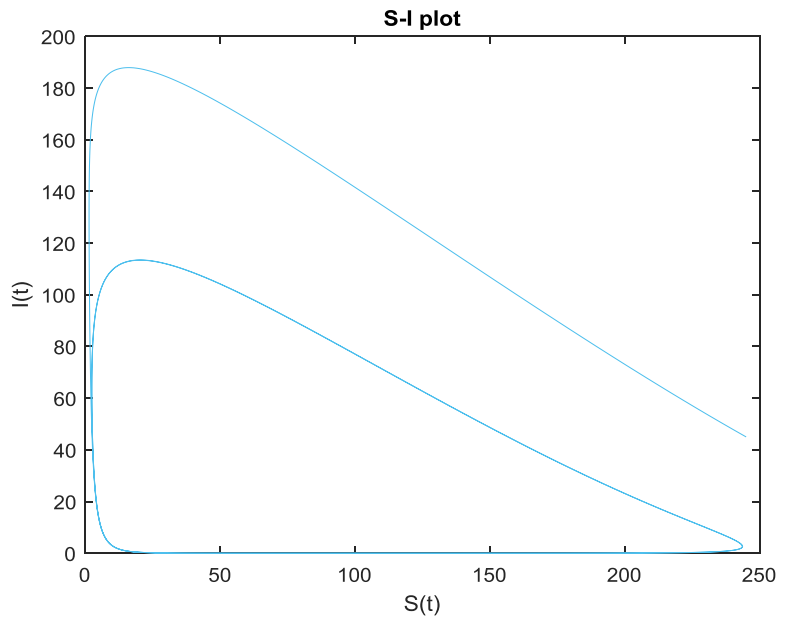

(a) $\mathrm{h}=0.01$

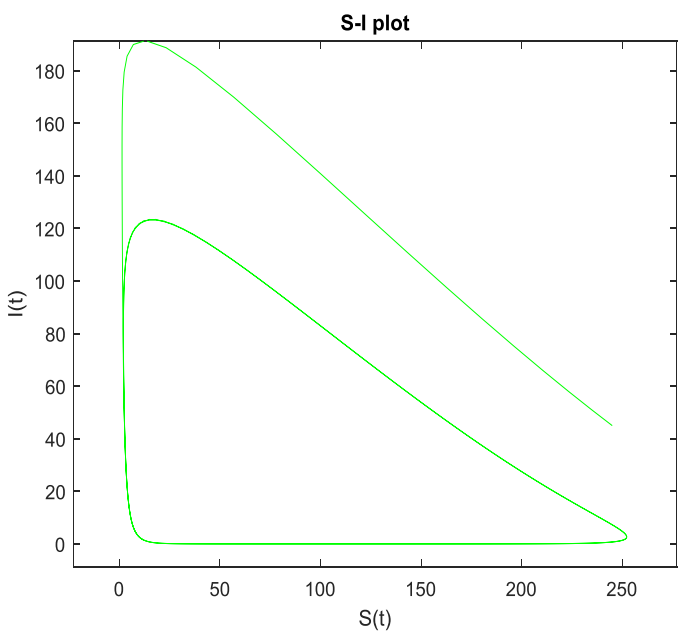

(b) $\mathrm{h}=0.1$

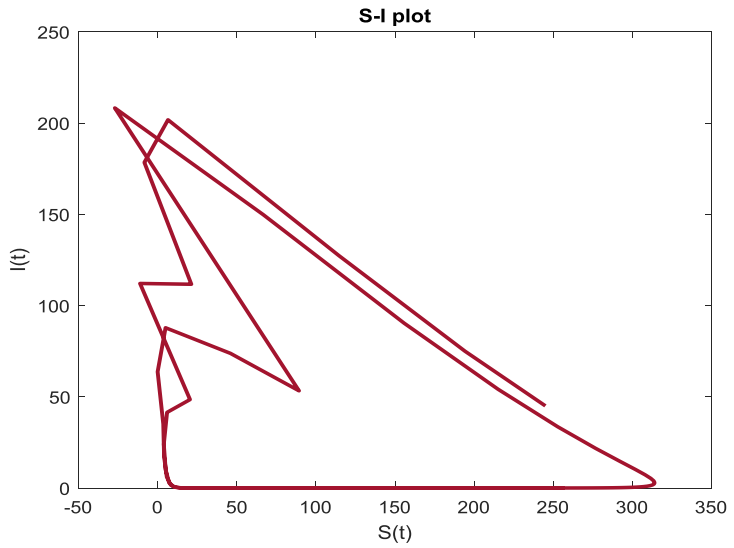

(c) $\mathrm{h}=0.5$

Fig $9 \mathrm{~S}-\mathrm{I}$ plot for $\alpha=0.06$

$A=7, \delta_{0}=0.002, \delta_{1}=0.005, \delta_{2}=0.01, a=2, b=0.02, \beta=0.02, \gamma=0.005$

at $(S, I, R)=(245,45,1)$. Here, we have represented the phase plane analysis of susceptible and infected population in Fig 8 and Fig 9, respectively. Fig 8 represents a stable limit cycle for $\alpha=0.08863$ (person) -1 (day) -1 when $h=0.01$ and 0.1 and a straight line when $\mathrm{h}=0.5$ and other parameters are same as given. Here no matter what the initial values of $\mathrm{S}(\mathrm{t})$ and $I(t)$, the populations eventually rise and fall periodically. Fig 8 (d) shows the variation of $h$. This isolated periodic trajectory is known as a stable limit cycle. Fig 9, trajectories represent phase portraits for $\alpha=0.06$ (person) -1 (day) -1 and different values of $h$ as well as other parameters are same as given.

\section{Conclusions}

The discrete epidemic model is more suitable to describe the spread of diseases since the epidemiological data are usually collected in discrete time units, such as daily, weekly, or monthly. In this study, we have studied the dynamical behavior of the discrete SIR epidemic model (2) with 
nonlinear incidence rate in presence of immunity. Sufficient conditions for the existence of the disease free equilibrium and endemic equilibria have been obtained. Model (2) is suitable to describe rotavirus, hand, foot, and mouth disease, influenza, chicken pox, measles, smallpox, mumps and SARS and so on which infectious diseases gains lifelong immunity. The discrete models are more appropriate forms than the continuous ones in order to directly fit the statistical data concerning infectious diseases. Motivated by the above facts, we have derived a discretized SIR epidemic model (1) by using Euler method. Therefore, the discretization scheme have the same equilibria and preserves the asymptotic stability of the equilibria for corresponding continuous models (1) in [1] as well as positivity and boundedness of the solutions. It is explained that the discrete model is dynamically balanced with its continuous model only for small step-size h, for large h, discrete model may exhibit complex dynamical behaviors. From numerical simulation, we see that for large size of $h$ discrete model is bifurcating most. The discrete model exhibits variant and rich dynamical behavior. Finally we can say, discrete model is more useful in epidemic model.

\section{Future Work}

We have no interest for future research in this topics.

\section{References}

[1] B. Dubey, P. Dubey, and U. S. Dubey, "Dynamics of an SIR model with nonlinear incidence and treatment rate", Appl. Appl. Math., 10: 718 - 737, December 2015.

[2] K. Dietz and J.A.P. Heesterbeek. Daniel Bernoulli's epidemiological model revisited. Mathematical Biosciences, 180: 1-21, 2002.

[3] R. Ross. The Prevention of Malaria. Dutton, New York, 2nd edition, 1911.

[4] W. O. Kermack and A. G. McKendrick. Contributions to the mathematical theory of epidemics. I. Proceedings of the Royal society of London. Series A, 115: 700-721, 1927. Reprinted in Bull. Math. Biol. 53 33-55, 1991.

[5] W. O. Kermack and A. G. McKendrick. Contributions to the mathematical theory of epidemics. II. The problem of endemicity. Proceedings of the Royal society of London, 138(834): 55-83, 1932.

[6] W. O. Kermack and A. G. McKendrick. Contributions to the mathematical theory of epidemics. III. Further studies of the problem of endemicity. Proceedings of the Royal Society of London, 141(843):94-122, 1933. Reprinted in Bull. Math. Biol. 53: 89-118, 1991.

[7] Y. Zhou, Z. Ma, and F. Brauer. "A discrete epidemic model for SARS transmission and control in China". Mathematical and Computer Modelling, 40(13): 1491 - 1506, 2004.
[8] L.J.S. Allen, M.A. Jones, and C.F. Martin. “A discrete-time model with vaccination for a measles epidemic". Mathematical Biosciences, 105(1): 111 - 131, 1991.

[9] H. Cao and Y. Zhou. "The discrete agestructured SEIT model with application to tuberculosis transmission in China". Mathematical and Computer Modelling, 55(34): 385-395, 2012.

[10] L.J.S. Allen and P. van den Driessche. "The basic reproduction number in some discretetime epidemic models". Journal of Difference Equations and Applications, 14(10-11): 11271147, 2008.

[11] C.L. Wesley, L.J.S. Allen, C.B. Jonsson, Y.K. Chu, and R.D. Owen. "A discretetime rodent-hantavirus model structured by infection and developmental stages". Advanced Studies in Pure Mathematics, 53: 1-12, 2009.

[12] Y. Luo, S. Gao, D. Xie, and Y. Dai. "A discrete plant disease model with roguing and replanting". Advances in Difference Equations, 2015(1), 2015.

[13] L. J.S. Allen and P. van den Driessche. "Relations between deterministic and stochastic thresholds for disease extinction in continuous- and discrete-time infectious disease models". Mathematical Biosciences, 2013.

[14] J. Zhang and Z. Jin. "Discrete time SI and SIS epidemic models with vertical transmission". Journal of Biological Systems, 17(2): $201-212,2009$.

[15] L. Pellis, N. M. Ferguson, and C. Fraser. "The relationship between real-time and discrete-generation models of epidemic spread". Mathematical Biosciences, 216(1): 63-70, 2008.

[16] D. Ludwig. "Final size distribution for epidemics". Mathematical Biosciences, 23(1): $33-46,1975$.

[17] M. H. A. Biswas, "On the Evaluation of AIDS Treatment: An Optimal Control Approach", Current HIV Research, 12(1): 112, 2014.

[18] M. H. A. Biswas, M.A.Islam, S. Akter, S. Mondal, M. S. Khatun, S.A.Samad, A. K. Paul and M. R. Khatun, "Modelling the Effect of Self-Immunity and the Impacts of Asymptomatic and Symptomatic Individuals on COVID-19 Outbreak", CMES-Computer Modeling in Engineering \& Sciences, 125(3): 1033-1060, 2020. 
[19] Li, L., Sun, G., Jin, Z. "Bifurcation and chaos in an epidemic model with nonlinear incidence rates". Appl. Math. Comput. 216: 1226-1234, 2010.

[20] Y.C Zhou, Z.E Ma."Global stability of a class of discrete age-structured SIS models with immigration". Math. Biosci. Eng. 6: 409-425, 2009.

[21] H. Z. Teng, Z.D. H. Jiang. "Stability analysis in a class of discrete SIRS epidemic models". Nonlinear Anal., Real World Appl. 13(5): 2017-2033, 2012.

[22] M. A. Mikucki, "Sensitivity analysis of the basic reproduction number and other quantities for infectious disease models",M. thesis, Colorado State University Fort Collins, Colorado Spring, 2012.

[23] P. Das, D. Mukherjee, and A. Sarkar. "Study of an S-I epidemic model with nonlinear incidence rate: discrete and stochastic version", Applied Mathematics and Computation 218(6): 2509 - 2515, 2011.

[24] A.-E. A. Elsadany, H. A. EL-Metwally, E. M. Elabbasy, and H. N. Agiza. 2012. "Chaos and bifurcation of a nonlinear discrete preypredator system", Computational Ecology and Software 2: 169-180.

[25] Z. Hu, Z. Teng, and H. Jiang.. "Stability analysis in a class of discrete SIRS epidemic models", Nonlinear Analysis: Real World Applications 13(5): 2017 - 2033, 2012.

[26] A. G. M. Selvam, R. Janagaraj and D. J. Praveen, "Behavior of a Discrete SIR Epidemic Model", American International Journal of Research in Science, Technology, Engineering \& Mathematics, ISSN (Print): 2328-3491, ISSN (Online): 2328-3580, ISSN (CD-ROM): 2328-3629.

[27] Z. Hu, Z. Teng and L. Zhang, "Stability and Bifurcation Analysis in a Discrete SIR Epidemic Model", Mathematics and Computer Simulations, 97: 80-93, 2014.

[28] A. Agrawal , A. Tenguria, G. Modi , "Stability Analysis of an Sir Epidemic Model with Specific Nonliner Incidence Rate", Mathematical Theory and Modeling, 6(1): 4551, 2016.
[29] Y. Xue and X. Duan, "Dynamic Analysis of an Sir Epidemic Model with Nonlinear Incidence Rate and Double Delays", International Journal of Information and Systems Sciences, 7(1): 92-102, 2010.

[30] O. Zakary1, M. Rachik1, I. Elmouki1, "On the Analysis of a Multi-Regions Discrete SIR epidemic model: an optimal control approach", Int. J. Dynam. Control, 5: 917-930, 2017.

[31] H. Cao, H. Wu and X. Wang. "Bifurcation analysis of a discrete SIR epidemic model with constant recovery", Advances in Difference Equations, 2020.

[32] M. H. A. Biswas, "Optimal Control of Nipa Virus Infections A Bangladesh Scenario", Pure and Applied Mathematics Advances and Applications, 12(1): 77-104, 2014.

[33] M. H. Kabir, M. O. Gani, S. Mandal and M. H. A. Biswas, "Modeling the dispersal effect to reduce the infection of COVID-19 in Bangladesh", Sensors International, 1, 2020.

[34] W. Chinviriyasit, S. Janreung, and S. Chinviriyasit, "On the backward bifurcation of an SEIRS epidemic model with nonlinear incidence rate", WSEAS TRANSACTIONS on SYSTEMS, 18, 2018.

[35] K. Henneman, D. V. Peursem, V. C. Huber, "Mathematical modeling of influenza and a secondary bacterial infection", WSEAS TRANSACTIONS on BIOLOGY and BIOMEDICINE, 10,2013.

\section{Creative Commons Attribution License 4.0 (Attribution 4.0 International, CC BY 4.0)}

This article is published under the terms of the Creative Commons Attribution License 4.0 https://creativecommons.org/licenses/by/4.0/deed.en_US 